\title{
Conformal Heating Using Scanned 1-D Phased Array for External Ultrasound Hyperthermia
}

\author{
Kuen-Cheng Ju', Yung-Yaw Chen', Win-Li Lin'², Te-Son Kuo' \\ ${ }^{1}$ Department of Electrical Engineering, National Taiwan University, Taipei, Taiwan \\ ${ }^{2}$ Institute of Biomedical Engineering, National Taiwan University, Taipei, Taiwan
}

Abstract-The feasibility of one-dimensional phased array transducer with mechanical scanning to produce a conformal heating for external ultrasound hyperthermia was evaluated. For the configuration of this system, numbers of focal spots were distributed within the target volume. To achieve the goal of conformal heating, an algorithm was introduced to calculate the power weighting for each field pattern to produce a heating volume conformal to the shape of the target volume. Simulation results showed that rather high cure rate in target volume with less damage rate in normal tissue can be obtained for target volumes with different shapes.

\section{INTRODUCTION}

The clinical goal of hyperthermia is to heat a target volume to therapeutic temperature $\left(43^{\circ} \mathrm{C}\right.$ in this study) conformal to the shape of a tumor and to avoid over-heating of normal tissues with appropriate ultrasound power deposition. However, during the heating process, portions of energy will be absorbed along the penetration path, which results in over-heating of normal tissues.

Focused scanned ultrasound system [1] can achieve precise control of localized heating patterns. However, a rather complex multi-degree mechanical motion is needed. Phased array transducer [2] has a potential advantage of steering the ultrasound energy without mechanical movement of transducers. Owing to some technical issues, the dimension of phased array transducer is limited, and the curable region is confined. Hybrid techniques that combine phased array transducer and mechanical scanning maybe a solution for this problem.

In this study, we undertook the development a one-dimensional ultrasound phased array transducer with mechanical scanning that could provide good conformability of tumor heating. The transducer system is capable of dynamically focusing on a target plane with numbers of focusing spots. For the purpose of conformal heating, the power deposition of the treatment area is constructed by proper combinations of local focal spots areas. An iterative gradient decent method is used to find an optimal weighting parameter set for this nonnegative constrained problem. Different shapes of tumors were tested, and the results show acceptable conformal heating effect in ultrasound hyperthermia.

\section{METHODOLOGY}

\section{System description}

A schematic diagram of scanned phased array transducer system is shown in Fig. 1, which consists of a one-dimensional phased array transducer and two mechanical driving systems, i.e. parallel scanning and rotating. The transducer array is attached to the scanning shaft, and is able to movie freely about the shaft. By appropriately driving signals of transducer array, it is capable of dynamically focus on $X^{\prime}-Z^{\prime}$ plane. Combining mechanical scanning and electrical focusing, target volume is covered with focal spots. However, owing to the cumulative power in the intervening region between skin and target volume, the undesired heating is produced. A solution to this problem is to increase the acoustic window [3]. For this reason, scanning shaft is rotated with an angle $\theta$ [Fig. 1(a)], then ultrasound energy is overlapped on the target volume and undesired cumulative power relatively is reduced.

\section{Ultrasound field calculation}

The acoustic power deposition pattern can be 
obtained from

$$
Q=\frac{\alpha p^{2}}{\rho c}
$$

where $Q$ is the absorbed power in tissue in $\mathrm{W} / \mathrm{m}^{3}$, absorption coefficient $(\alpha)$ is $5(\mathrm{~Np} / \mathrm{m} / \mathrm{MHz})$, tissue density $(\rho)$ is $1000\left(\mathrm{~kg} / \mathrm{m}^{3}\right)$, and $p$ is the ultrasonic pressure field. The ultrasound pressure field calculation is based on Rayleigh-Sommerfeld diffraction theory. The calculation of each focused pattern was conducted by an iterative pseudo inverse method [4] to obtain uniform amplitude driving signals, and to increase the efficiency of the transducer array.

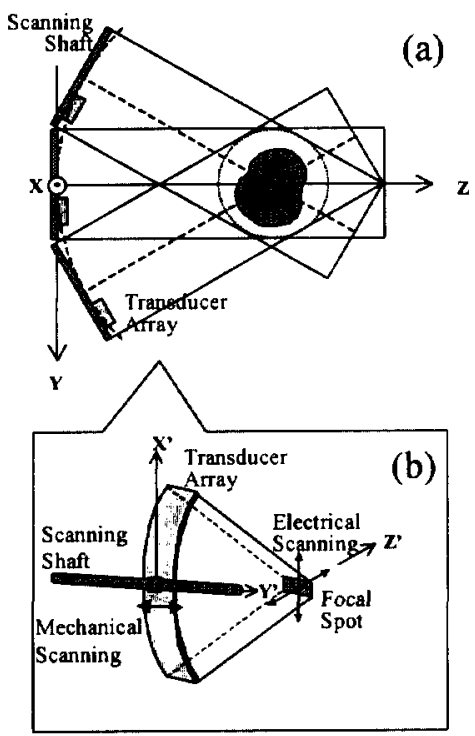

Fig. 1. Schematic diagram of the scanned phased array transducer system

\section{Thermal model}

Heat transfer calculation is performed by bioheat transfer equation (BHTE),

$$
\rho c_{1} \frac{\partial T}{\partial t}=k \nabla^{2} T-w c_{b}\left(T-T_{b}\right)+Q .
$$

Thermal conductivity $(k)$ is $0.5(\mathrm{~m} / \mathrm{s})$, the specific heat in tissue $\left(c_{t}\right)$ and blood $\left(c_{b}\right)$ are both $3770\left(\mathrm{~J} / \mathrm{kg}^{\circ} \mathrm{C}\right)$, the blood temperature $\left(T_{b}\right)$ is $37{ }^{\circ} \mathrm{C}$, and the blood perfusion $\left(w_{b}\right)$ is $5\left(\mathrm{~kg} / \mathrm{m}^{3} \mathrm{~s}\right)$. The power deposition patterns $(Q)$ obtained from Eq. (1) is substituted into BHTE, and 3-D finite difference technique is used to find the solution of this equation.

\section{Scanning Model}

Because of the large thermal time constant, the temperature response from a set of scanning field patterns can be approximated as that from a spatial-temporal averaged field when scanning speed is rather high. Furthermore, McGough et al. [5] demonstrate that owing to the linearity property of BHTE, the spatial superposition of a set of temperature response from multiple focal points approximates the whole temperature field. Hence, the approximate temperature response produced by a scanning system can be given by $\boldsymbol{T}_{\mathrm{M} \times 1}=\boldsymbol{B}_{\mathrm{M} \times \mathrm{N}} \boldsymbol{W}_{\mathrm{N} \times 1}$. $\boldsymbol{T}$ is a $\mathrm{M} \times 1$ vector describe the temperature response at $\mathrm{M}$ discrete field points. $\boldsymbol{B}$ is temperature elevation matrix describe temperature raise produced by $\mathrm{N}$ field patterns, and $\boldsymbol{W}$ is the weighting vector of $\mathrm{N}$ field patterns.

\section{Iterative Gradient Decent Optimization}

The goal of optimization is to find an appropriate weighting vector $\boldsymbol{W}$ to elevate the temperature within target volume to therapeutic level and reduce damage rate to normal tissue. Because of the impossibility of negative ultrasound energy, analytic technique such as pseudo inverse method cannot be used to find the optimal solution. In this study, an iterative gradient-based optimization with penalty function [6] was used to find an optimal solution for this nonnegative-constrained problem. We define the cost function as

$$
\begin{aligned}
& \text { Cost }=\sum_{i=1}^{M}\left(T_{D, i}-T_{i}\right)+K \cdot \sum_{j=1}^{N} f\left(w_{j}\right) \\
& f\left(w_{j}\right)=\left\{\begin{array}{cl}
0 & , w_{j} \geq 0 \\
w_{j}^{2} & , w_{j}<0
\end{array}\right.
\end{aligned}
$$

where $T_{D}$ is the desired temperature distribution, $f\left(w_{j}\right)$ is nonnegative quadratic penalty and $K$ is a constant, which is used to emphasize the penalty. The iterative algorithm to find optimal weighting set is

$$
\begin{aligned}
w_{i}^{i+1}= & w_{i}^{t}+\Delta w_{i}^{\prime} \\
\Delta w_{t}= & -\frac{\partial \operatorname{Cos} t}{\partial w_{i}}=-\sum_{j=1}^{M} \frac{\partial \operatorname{Cost}}{\partial T_{j}} \cdot \frac{\partial T_{j}}{\partial w_{i}}-K \frac{\partial f\left(w_{i}\right)}{\partial w_{i}} \\
& =2 \sum_{j=1}^{M}\left(T_{D, j}-T_{j}\right) \cdot b_{j i}-K f^{\prime}\left(w_{i}\right) \quad, i=1 \ldots N
\end{aligned}
$$

where $b_{i j}$ is the element in matrix $\boldsymbol{B}$, and $\boldsymbol{t}$ labels the iteration number. 


\section{RESULTS and DISCUSSION}

The one-dimensional phased array in this system was simulated at an operating frequency of $1 \mathrm{MHz}$. The one-dimensional phased array consists of 64 elements, each $3 \mathrm{~mm}$ in height and $20 \mathrm{~mm}$ in width, with radius of curvature of $18 \mathrm{~cm}$. The ultrasound field model was a simple water-tissue medium. The center of the tumor was located at depth $10 \mathrm{~cm}$ from the water-tissue interface and $16 \mathrm{~cm}$ from center of transducer array.

\section{Focal Spot Distribution}

Fig. 2 shows the placement of focal spots in each elevation plane for each rotate angle. The focal layers are distributed from $-2 \mathrm{~cm}$ to $+2 \mathrm{~cm}$ with gaps of 0.5 $\mathrm{cm}$. The scanning shaft was rotated with angle of -30 , $0^{\circ}$ and $+30^{\circ}$. In each rotate angle, 47 focal spots were arranged in a focal layer and were distributed form $13 \mathrm{~cm}$ to $19 \mathrm{~cm}$ in Z'-direction and form $-3 \mathrm{~cm}$ to $+3 \mathrm{~cm}$ in $Y^{\prime}$-direction with space of $1 \mathrm{~cm}$. Not all of these focal spots will be used in a treatment, but properly pre-selected. Weightings of selected focal spots were substituted into the optimization algorithm to find an optimal weighting set.

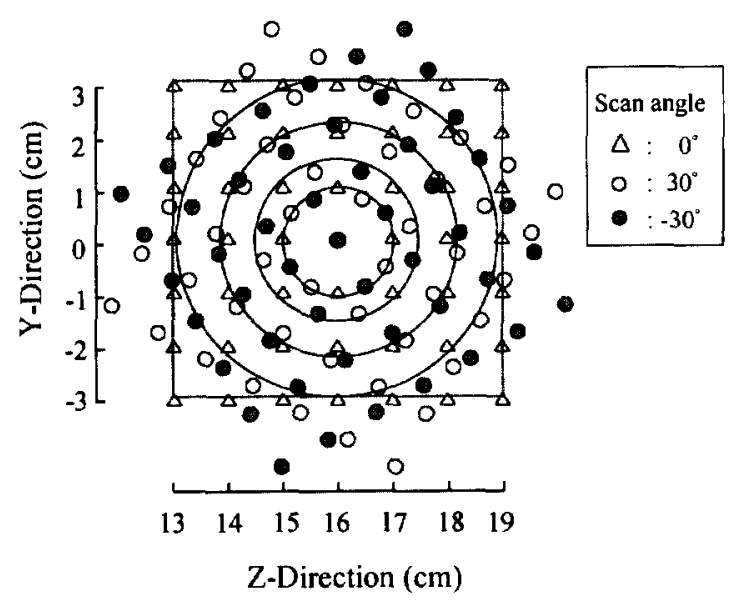

Fig. 2 The displacements of focal spots in each focal layer in $\mathrm{X}$ -plane. In each rotate angle, focal spots distributed from $13 \mathrm{~cm}$ to $19 \mathrm{~cm}$ in $Z^{\prime}$-direction and $-3 \mathrm{~cm}$ to $+3 \mathrm{~cm}$ in $Y^{\prime}$-direction with space of $1 \mathrm{~cm}$.

\section{Cylindrical Heating Volume}

Owing to the concentric distribution of focal points, a cylindrical desired heating volume was tested. In this simulation, the cylindrical target volume has radius of
$2 \mathrm{~cm}$ and height of $4 \mathrm{~cm}$, and only 5 focal layers (X = $0, \pm 1$, and $\pm 2 \mathrm{~cm}$ ) are used. Fig. 3 show the resulting temperature distributions in $X=0$ plane [Fig. 3(a)] and $Y=0$ plane [Fig. 3(b)]. It can be seen that the therapeutic temperature of $43^{\circ} \mathrm{C}$ contour (shaded area) almost covered the desired heating region (dotted line area), although a portion of normal tissue is damaged. Fig. 3(c) shows the $3-\mathrm{D} 43^{\circ} \mathrm{C}$ isotherm plot. Compare to the size of focal spots, the gaps of each focal layer is large. Higher ultrasound power of each focal layer is needed to elevate the temperature of tissue between each focal layer to the therapeutic level by heat conduction. Hence, the rugged isotherm plot in Fig. 3 (c) shows the good correspondence to the placement of focal layers. Fig. 3(d) shows the cure rate (CR) in target volume and damage rate (DR) in normal tissue to evaluate the conformability of this heating strategy. The definition of cure rate is the fraction of target volume with temperature equal to or higher than $43^{\circ} \mathrm{C}$, and damage rate is defined as the ratio of volume in normal tissue with temperature equal to or higher than $43^{\circ} \mathrm{C}$ to the volume of target volume. Furthermore, the conformal index $(\mathrm{CI})$, defined as cure rate minus damage rate, was $84.8 \%$. The peak temperature occurs inside the tumor at $44.5^{\circ} \mathrm{C}$, which shows a good uniformity in temperature distribution.
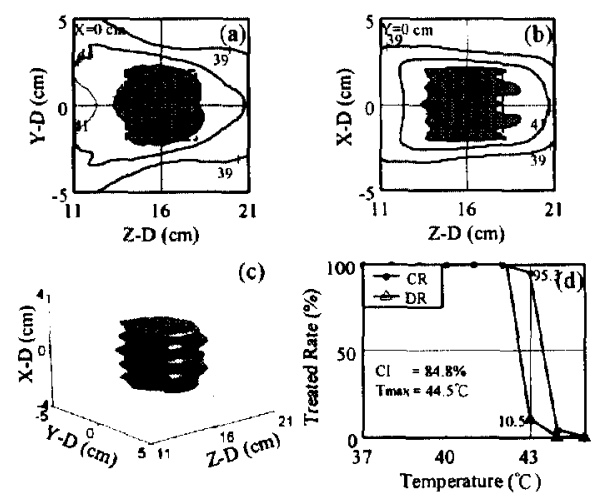

Fig. 3 Results of a $2 \mathrm{~cm}$ radius and $4 \mathrm{~cm}$ high cylindrical target volume produced by the scanned phased array system in (a) $\mathrm{X}=0$ plane, (b) $\mathrm{Y}=0$ plane. (c) shows $3-\mathrm{D} 43{ }^{\circ} \mathrm{C}$ isotherm surface plot, and (d) displays the quantitative indices of the resulting heating patterns.

\section{Other shaped Heating Volume}

In Fig. 4(a), the target volume is a $4 \times 4 \times 4 \mathrm{~cm}^{3}$ cube. Dotted lines present the boundaries of the target volume. In comparison, totally nine focal layers $(X=$ 
$0, \pm 0.5, \pm 1.0, \pm 1.5$ and $\pm 2.0 \mathrm{~cm})$ were used, and a smoother resulting heating pattern was obtained.

Fig. 4(c)-(d) show results of $4 \times 2 \times 2 \mathrm{~cm}^{3}, 2 \times 4 \times 2$ $\mathrm{cm}^{3}$ and $2 \times 2 \times 4 \mathrm{~cm}^{3}$ rectangular target volume, respectively. Table I summarize the results of target volumes with different shapes tested in this study. It shows the non-uniformity of temperature distribution in Fig. $4(\mathrm{~d})$. The maximum temperature of $48.3^{\circ} \mathrm{C}$ was obtained. One of the reasons can be the smaller effective acoustic window, which result in considerable ultrasound energy accumulated within the target volume. For the same reason, the situation of Fig. 4(b) presents a terrible damage rate of $23 \%$, owing to extra-undesired ultrasound energy accumulated outside the target volume.
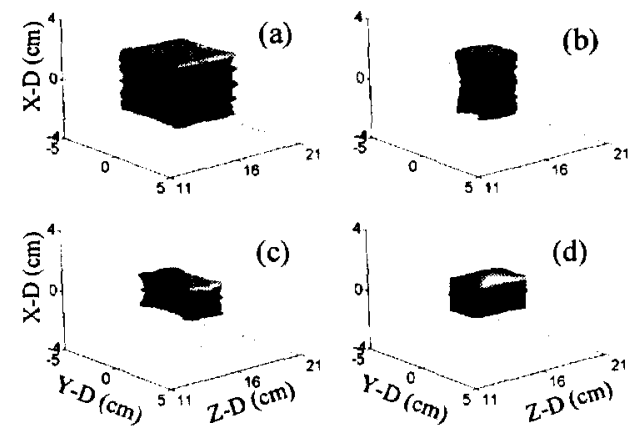

Fig. $443^{\circ} \mathrm{C}$ isotherm plots of (a) $4 \times 4 \times 4 \mathrm{~cm}^{3}$ cube, (b) $4 \times 2 \times 2$ $\mathrm{cm}^{3}$ rectangular, (c) $2 \times 4 \times 2 \mathrm{~cm}^{3}$ rectangular and (d) $2 \times 2 \times 4 \mathrm{~cm}^{3}$ rectangular target volume produced by the scanned phased array

\section{CONCLUSION}

In this study, we developed an ultrasound hyperthermia applicator to produce conformal heating. Different shapes of tumors were tested. Simulation results show that in most cases the percentage for successful heating is over $80 \%$ of the target tumor volume and the normal tissue damage rate was less than $20 \%$ of the target tumor volume. Comparing to the existing studies, this method seems to provide an acceptable conformal heating effect in ultrasound hyperthermia. The idea of combining the techniques of electrical focusing
TABLE I Heating results for different target volume

\begin{tabular}{|l|c|c|c|c|c|}
\hline Shapes (cm) & $\begin{array}{c}\text { Numbers of } \\
\text { focal spots }\end{array}$ & $\begin{array}{c}\text { Cure } \\
\text { Rate(\%) }\end{array}$ & $\begin{array}{c}\text { Damage } \\
\text { Rate(\%) }\end{array}$ & $\begin{array}{c}\text { Conformal } \\
\text { Index }\end{array}$ & $\begin{array}{c}\mathrm{T}_{\max } \\
\left({ }^{\circ} \mathrm{C}\right)\end{array}$ \\
\hline $\begin{array}{l}\text { R=2, H=4 } \\
\text { Cylinder }\end{array}$ & 81 & 95.3 & 10.5 & 84.8 & 44.5 \\
\hline $\begin{array}{l}4 \times 4 \times 4 \\
\text { Cube }\end{array}$ & 92 & 95.4 & 11.6 & 83.8 & 45.1 \\
\hline $\begin{array}{l}4 \times 2 \times 2 \\
\text { Rectangular }\end{array}$ & 37 & 94.3 & 23.0 & 71.3 & 44.0 \\
\hline $\begin{array}{l}2 \times 4 \times 2 \\
\text { Rectangular }\end{array}$ & 51 & 89.9 & 6.2 & 83.7 & 43.7 \\
\hline $\begin{array}{l}2 \times 2 \times 4 \\
\text { Rectangular }\end{array}$ & 35 & 95.1 & 15.7 & 79.4 & 48.3 \\
\hline
\end{tabular}

and mechanical scanning has the advantages of enlarging the acoustic window and providing dynamical focusing ability, which are the essence of successful conformability.

\section{REFERENCES}

[1] E. S. Ebbini and C. A. Cain, "A spherical-section ultrasound phased array applicator for deep localized hyperthermia. Uitrason. Ferroelect. Freq. Contr., vol. 38, no. 7, pp. 634643, 1991

[2] E. S. Ebbini and C. A. Cain, "Multiple-focused ultrasound phased-array pattern synthesis: optimal driving-signal distributions for hyperthermia," IEEE Trans. Ultrason. Ferroelect. Freq. Contr., vol. 36, no. 5, pp. 540-548, 1989

[3] W. L. Lin, et al., "Relationship between acoustic aperture size and tumor condition for external ultrasound hyperthermia," Medical physics, vol.26, pp. 818-824, 1999

[4] R. J. McGough et al., "Direct computation of ultrasound phased-array driving signals from a specified temperature distribution for hyperthermia," IEEE Trans. Biomed. Eng., vol. 39 , no. 8, pp. 825-835, 1992

[5] B. S. Gottfried, J. Weisman, "Introduction to optimization theory," New Jersey: Prentice-Hall, 1973

[6] K. Hynynen, et al., "A scanned focused multiple transducer ultrasonic system for localized hyperthermia treatments," Int. J. hyperthermia, vol. 3, pp. 21-35, 1987 